\title{
Medial prefrontal lesions in mice impair sustained attention but spare maintenance of information in working memory
}

\author{
Julia B. Kahn, ${ }^{1}$ Ryan D. Ward, ${ }^{2}$ Lora W. Kahn, ${ }^{2,6,8}$ Nicole M. Rudy, ${ }^{5}$ Eric R. Kandel, ${ }^{1,2,5,6,7}$ \\ Peter D. Balsam, ${ }^{1,3,4}$ and Eleanor H. Simpson ${ }^{1,5,9}$ \\ ${ }^{1}$ Department of Psychiatry, Columbia University, New York, New York 10032, USA; ${ }^{2}$ Department of Neuroscience, Columbia \\ University, New York, New York 10032, USA; ${ }^{3}$ Department of Psychology, Columbia University, New York, New York 10027, USA; \\ ${ }^{4}$ Department of Psychology, Barnard College, New York, New York 10027, USA; ${ }^{5}$ New York State Psychiatric Institute, New York, New \\ York 10032, USA; ${ }^{6}$ Howard Hughes Medical Institute, Columbia University, New York, New York 10032, USA; ${ }^{7}$ Kavli Institute for Brain \\ Science, College of Physicians and Surgeons of Columbia University, New York, New York 10032, USA
}

\begin{abstract}
Working memory and attention are complex cognitive functions that are disrupted in several neuropsychiatric disorders. Mouse models of such human diseases are commonly subjected to maze-based tests that can neither distinguish between these cognitive functions nor isolate specific aspects of either function. Here, we have adapted a simple visual discrimination task, and by varying only the timing of events within the same task construct, we are able to measure independently the behavioral response to increasing attentional demand and increasing length of time that information must be maintained in working memory. We determined that $\mathrm{mPFC}$ lesions in mice impair attention but not working memory maintenance.
\end{abstract}

[Supplemental material is available for this article.]

A deficit in working memory, the ability to actively maintain and manipulate information over short time intervals (Baddeley 1992), is a core cognitive symptom of schizophrenia (Park and Holzman 1992; Barch and Smith 2008) and depression (Harvey et al. 2004; Rose and Ebmeier 2006). Working memory is a complex construct that involves a number of discrete psychological processes, including the maintenance of information, flexible updating and manipulation, and sensitivity to interference, and it is strongly influenced by attentional processes (see the NIMH Research Domain Criteria working memory workshop proceedings [Research Domain Criteria Project 2010] and Awh and Jonides 2001). Because the neural substrates for each of these discrete processes are likely to be different, each component must be precisely isolated at both the behavioral and neural levels of analysis in order to relate behavioral and biological processes.

Mouse molecular models involving genetic and virally mediated manipulations, including optogenetics, can provide powerful tools for identifying the cellular and molecular mechanisms that support cognitive functions such as working memory. However, the value of such cross-species translational research depends upon the existence of functional homology and the availability of accurate behavioral assays to isolate discrete cognitive processes in mice (see Ward et al. 2011 for discussion). Researchers have identified the medial prefrontal cortex (mPFC), consisting of the infralimbic, prelimbic, and anterior cingulate cortices, as the rodent brain structure homologous to the primate dorsal lateral PFC (DLPFC) due to the similarity of anatomical connections (Furster 1997; Ongur and Price 2000).

${ }^{8}$ Present address: Department of Neurosurgery, Tulane University, New Orleans, LA 70112, USA

${ }^{9}$ Corresponding author

E-mail es534@columbia.edu

Article is online at http://www.learnmem.org/cgi/doi/10.1101/lm.026302.112.
Several rodent studies have shown that mPFC damage results in poor performance in working memory tasks (Brito and Brito 1990; Granon et al. 1994; Seamans et al. 1995; Ragozzino et al. 1998; Taylor et al. 2003). The majority of these studies used maze-based tasks, such as delayed alternation or delayed match or nonmatch to sample paradigms. Such maze-based tasks do not isolate the individual cognitive processes that are involved in solving working memory tasks, such as acquisition of the task rules, maintenance of information over the delay, flexible updating, or resistance to interference, because the contribution of each of these processes is assayed together within the same trials. Moreover, poor performance in such tasks may result from disruption of processes outside of working memory, such as attention, motivation, associative learning, or differences in the tendency to perseverate. Consequently, altered performance in such maze-based tasks cannot be attributed conclusively to a disturbance in any specific cognitive function.

In an early maze-alternative approach to study working memory, lesion of the $\mathrm{mPFC}$ in rats was reported to impair performance on an operant delayed non-match to place (DNMTP) task (Aggleton et al. 1995). However, performance was affected at all delay intervals tested, including the 0 delay condition. Therefore, poor performance was due to the disruption of a process or processes other than maintaining the information over the delay. Indeed, an increase in bias was observed in the mPFC-lesion group. Since this early study, a better understanding of the cognitive deficits produced in rats with lesions or with dopaminergic or cholinergic perturbations of the PFC has been achieved using other operant-based paradigms. This work has been comprehensively reviewed elsewhere (Dalley et al. 2004; Chudasama 2011). One such operant-based paradigm, the combined attention and memory (CAM) task was designed to isolate attentional from memory processes in rats within individual trials (Chudasama and Robbins 
2004). Unfortunately, the training alone for this task takes up to 6 mo, making its use in mice problematic, as the animals may be subject to the effects of aging during testing, particularly if multiple conditions are to be tested within subjects.

One effective strategy to isolate working memory maintenance and attention would be to assay these discrete processes in procedures that are equivalent with respect to stimuli, motivation, required response, and overall task structure (see Ward et al. 2012a for discussion). Here, we describe such an approach using variants of a two-choice visual discrimination paradigm. By manipulating only the timing of events within the same procedure, we were able to assay sustained attention separately from working memory maintenance and have determined the impact of mPFC lesions in mice on each of these cognitive functions.

In this study, we performed lesion and sham surgeries in the mPFC (anterior cingulate, prelimbic, and infralimbic cortices) of adult mice before they began training on a visual discrimination task. Specifically, animals were anesthetized with ketamine/ xylene, and $1.0 \mu \mathrm{L}$ of ibotenic acid $(5 \mathrm{mg} / \mathrm{mL}$ in saline) was stereotactically delivered to $+2.4 \mathrm{~mm}$ antero-posterior, $\pm 0.4 \mathrm{~mm}$ medio-lateral, from bregma and $-2.0 \mathrm{~mm}$ ventral from dura. Sham animals underwent the same surgical procedure, but saline was delivered instead of ibotenic acid. Behavioral training began after a 4-wk post-operative recovery period.

All training and testing sessions occurred once per day, $7 \mathrm{~d}$ per wk. Animals were first trained to consume evaporated milk from the liquid dipper, which was centrally located between two retractable levers in standard testing chambers (ENV307W, MedAssociates). The mice were then trained to press the lever to obtain rewards on a continuous reinforcement (CRF) schedule as described previously (Ward et al. 2012b). Each CRF session ended after 60 rewards or $60 \mathrm{~min}$, whichever occurred first. Subjects that had earned $<30$ rewards on the third day of CRF training were given an overnight (14-h) session with no limit on earned rewards. Subjects with $<20$ responses on the seventh day of CRF training were removed from the experiment (three lesion, one sham). Discrimination training then occurred in several phases. In all phases, each trial began with an intertrial interval (ITI) of unpredictable duration (mean $=45 \mathrm{sec}$, range $2.74-148.13 \mathrm{sec}$ ). During single light-single lever training, subjects received trials where a cue light above a lever on either the left or right side of the chamber was illuminated for $10 \mathrm{sec}$; $1 \mathrm{sec}$ after the cue's termination, the

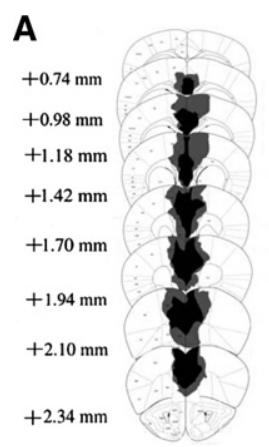

B

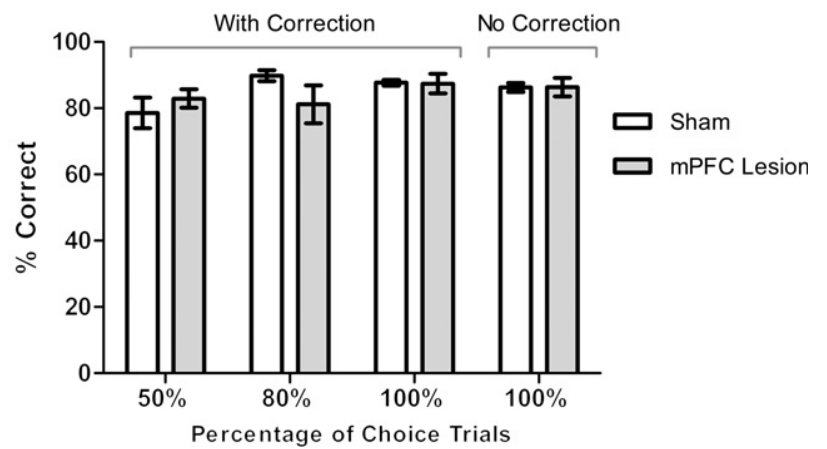

Figure 1. Lesion of the mouse mPFC does not impair acquisition of a visual discrimination task. (A) Schematic representation of the maximal (gray) and minimal (black) extent of lesion damage in the mPFC-lesion mice. Anterior position relative to bregma is provided for each section. (B) Groups' averaged percent correct responses during training with $50 \%$ choice trials (three sessions), $80 \%$ choice trials (three sessions), $100 \%$ choice trials (nine sessions), all with the correction procedure, and $100 \%$ choice trials without the correction procedure (12 sessions). Performance during each stage of training was unaffected by mPFC lesion. $n=7$ per group. lever beneath the cued light was presented for $10 \mathrm{sec}$. Pressing the lever beneath the cued light resulted in dipper reward. The cue light/lever position alternated daily across a total of four sessions, until the mice reliably pressed the lever after each stimulus cue presentation. During choice training, a percentage of the trials were single light-single lever trials as described above, while the remaining percentage were choice trials. The position of the cue light (left or right) was randomly determined from trial to trial. During choice trials, both of the levers were inserted $1 \mathrm{sec}$ after the cue's termination, and a response to the lever that had been cued at the beginning of the trial was rewarded. Incorrect responses resulted in a correction procedure, where the trial was repeated with the cue light in the same location until a correct response was made. Training consisted of three sessions with 50\% choice:50\% single lever-light trials, three sessions of $80 \%$ choice: $20 \%$ single leverlight trials, and nine sessions of $100 \%$ choice trials, all with correction. This was followed by 12 sessions of $100 \%$ choice trials without correction. The entire training period lasted $6 \mathrm{wk}$, after which sustained attention was assessed in $100 \%$ choice sessions (without correction) with varying cue light durations, presented in decreasing order (10 sec, $5 \mathrm{sec}, 2 \mathrm{sec}, 1 \mathrm{sec}, 0.5 \mathrm{sec}$, and $0.1 \mathrm{sec}$ ); each duration was tested for three sessions. Working memory maintenance was then assessed in $100 \%$ choice trials with a 5 -sec cue duration with delay intervals of increasing duration ( $2 \mathrm{sec}, 4 \mathrm{sec}, 8 \mathrm{sec}, 16$ $\mathrm{sec}, 24 \mathrm{sec}, 32 \mathrm{sec}$, and $40 \mathrm{sec}$ ) inserted between the cue offset and the lever presentation; each duration was tested for three sessions. All testing was completed in $6 \mathrm{wk}$. More detail of all training and testing is provided in Supplemental Material.

After completion of the behavioral study, mice were transcardially perfused with $4 \%$ PFA in PBS, their brains extracted, cryosectioned, thaw-mounted onto slides, stained with cresyl violet and examined microscopically. The minimum and maximum extent of the lesions on a series of coronal sections are depicted in Figure 1A. The lesions centered on the prelimbic cortex (PrL), extended ventrally to the infralimbic cortex (IL), and extended dorsally to include anterior portions of the cingulate cortex $(\mathrm{Cg} 1$ and Cg2). Mice with mPFC lesions acquired the visual discrimination task at the same rate as the sham-lesioned mice (Fig. 1B). A repeated-measures ANOVA revealed a significant main effect of session on performance across all phases of training $\left(F_{(26,312)}=1.98\right.$, $P=0.004)$ but no significant main effect of lesion $\left(F_{(1,12)}=0.65\right.$, $P=0.87)$ and no significant interaction between sessions and lesion $\left(F_{(26,312)}=1.38, P=0.104\right)$. A repeated-measures ANOVA across sessions within each phase of training revealed that neither session, lesion, nor an interaction between session and lesion had a significant effect on performance; $P$ values $>0.05$ for each phase. An mPFC lesion, therefore, had no significant impact on the acquisition of the visual discrimination procedure, allowing us to use the procedure to specifically assay sustained attention and working memory maintenance.

To assess sustained attention in our cohort of mPFC-lesioned mice, we varied the duration of stimulus cue presentation in our visual discrimination task. Figure $2 \mathrm{~A}$ shows that the mean percent of correct responses (averaged across three sessions) declined as cue duration decreased. A repeated-measures ANOVA revealed a significant main effect of both cue duration $\left(F_{(5,60)}=68.36, P<0.0001\right)$ and lesion $\left(F_{(1,12)}=7.31, P=0.019\right)$ and no 
A

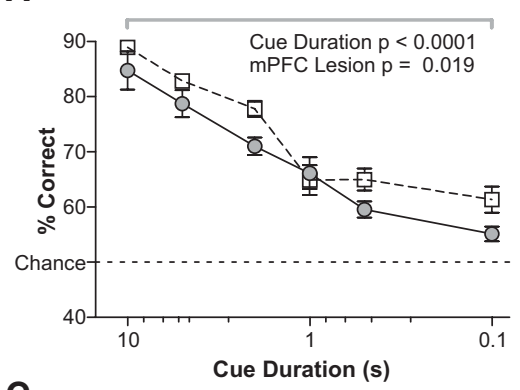

C

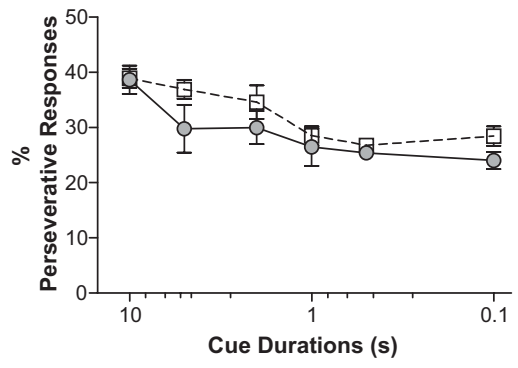

B

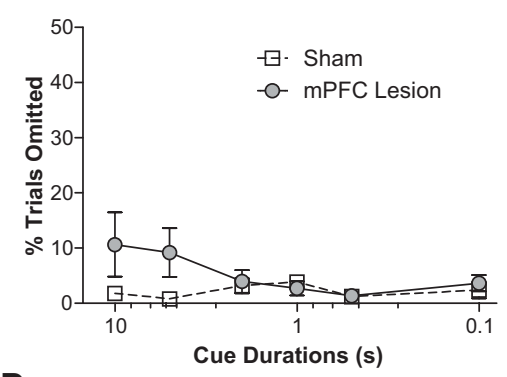

D

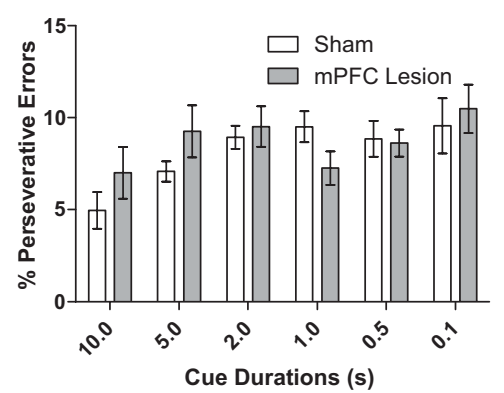

Figure 2. Lesion of the mouse mPFC impacts performance on a sustained attention task. mPFC lesion resulted in significantly lower accuracy $(A)$ but did not impact the percentage of trials in which no response was made (errors of omission, $B$ ). The percentage of trials in which the mice responded on the previously reinforced lever (percent perseverative responses, $C$ ) and the percentage of incorrect trials on which the mice responded on the previously reinforced lever (percent perseverative errors, $D$ ), averaged across three daily sessions for each cue duration, were not different between the groups. The $x$-axis in $A-C$ is on a reverse log 10 scale to best visualize the range of cue durations tested. $n=7$ per group.

significant interaction between cue duration and lesion $\left(F_{(5,60)}=\right.$ $1.49, P=0.207)$. These data are consistent with previous reports that the rat $\mathrm{mPFC}$ is required for accurate performance in a test of sustained attention (Passetti et al. 2002).

We analyzed several variables in the task to see if processes other than sustained attention were affected. We recorded latency to respond to determine if mPFC lesion affected general motor capacity. We assessed motivation by analyzing the latency to collect rewards, the number of earned rewards that were retrieved, and the number of trials in which the subject failed to respond. Mean response latencies increased as cue duration decreased but were not affected by lesion. There was a significant interaction between cue duration and lesion (Table 1); a Bonferroni post-test revealed no significant difference between sham and lesion response latency for any specific cue duration; all $P^{\prime} s>0.05$. The mean latency to collect earned rewards decreased as cue dura- tion decreased but was not affected by lesion. In this case, there was no significant interaction between cue duration and lesion (Table 1). Consistent with the fact that mPFC lesion does not affect latency to collect rewards, there was no significant effect on the percentage of earned rewards that were retrieved (Table 1). The number of trials in which the mice failed to make a response (\% trials omitted) was not affected by lesion or cue duration, but a significant interaction between these variables was observed (Fig. 2B). A Bonferroni post-test revealed no significant difference between the percentage of trials omitted by sham and lesion subjects for any specific cue duration; all $P$ 's $>0.05$.

Previously, it has been reported that mPFC lesions in rats can increase perseverative behavior, specifically when the cue duration is very short $(0.2 \mathrm{sec}$ or less) (Passetti et al. 2002); therefore, we determined the percentage of total responses that were made on the lever that was reinforced on the previous trial. Figure $2 \mathrm{C}$ shows no significant difference in the percentage of perseverative responses made by lesion and sham mice across the sessions, although a trend was observed (Table 1). To determine if the increase in errors made by the lesion group was a result of perseverative behavior, we determined the percentage of errors in which the mouse incorrectly responded on the lever that was reinforced in the previous trial. Again, there was no significant difference between the groups (Fig. 2D; Table 1).

To examine working memory maintenance, we used the 5 -sec cue duration condition and imposed delays of various lengths between the offset of the cue and the presentation of the choice levers. This cue duration was chosen because it resulted in performance at a high enough level of accuracy that any detrimental effects of increasing delay duration could be observed. Figure 3A illustrates the mean percent of correct responses made by lesion and sham mice, averaged across three daily sessions, as a function of delay duration. A repeated-measures ANOVA revealed a significant effect of delay duration $\left(F_{(6,72)}=74.85, P<\right.$ $0.0001)$ but no significant effect of lesion $\left(F_{(1,72)}=0, P=0.958\right)$ nor delay duration $\times$ lesion interaction $\left(F_{(6,72)}=0.3, P=0.912\right)$.

Table 1. Results of repeated-measures ANOVA for both the attention and working memory maintenance assays

\begin{tabular}{|c|c|c|c|c|c|c|c|c|c|c|c|c|}
\hline & \multicolumn{6}{|c|}{ Attention task } & \multicolumn{6}{|c|}{ Working memory } \\
\hline & \multicolumn{2}{|c|}{ mPFC lesion } & \multicolumn{2}{|c|}{ Cue duration } & \multicolumn{2}{|c|}{ Interaction } & \multicolumn{2}{|c|}{ mPFC lesion } & \multicolumn{2}{|c|}{ Delay duration } & \multicolumn{2}{|c|}{ Interaction } \\
\hline & $F_{(1,12)}$ & $P$ & $F_{(5,60)}$ & $P$ & $F_{(5,60)}$ & $P$ & $F_{(1,72)}$ & $P$ & $F_{(6,72)}$ & $P$ & $F_{(6,72)}$ & $P$ \\
\hline$\%$ Correct & 7.31 & $=0.019$ & 68.36 & $<0.0001$ & 1.49 & $=0.207$ & 0 & $=0.958$ & 74.85 & $<0.0001$ & 0.30 & $=0.912$ \\
\hline Response latency & 0.81 & $=0.385$ & 4.21 & $=0.002$ & 2.42 & $=0.046$ & 0.26 & $=0.622$ & 27.88 & $<0.0001$ & 0.66 & $=0.683$ \\
\hline Reward latency & 3.66 & $=0.080$ & 3.07 & $=0.016$ & 0.72 & $=0.612$ & 1.86 & $=0.198$ & 2.933 & $=0.013$ & 0.45 & $=0.840$ \\
\hline$\%$ Rewards retrieved & 1.00 & $=0.337$ & 1.00 & $=0.426$ & 1.00 & $=0.426$ & 0.35 & $=0.566$ & 2.443 & $=0.033$ & 2.73 & $=0.019$ \\
\hline$\%$ Trials omitted & 1.63 & $=0.226$ & 1.66 & $=0.158$ & 2.77 & $=0.03$ & 1.15 & $=0.305$ & 1.628 & $=0.152$ & 0.95 & $=0.467$ \\
\hline$\%$ Perseverative responses & 3.15 & $=0.100$ & 9.80 & $<0.0001$ & 0.62 & $=0.68$ & 0.26 & $=0.622$ & 22.12 & $<0.0001$ & 0.42 & $=0.865$ \\
\hline$\%$ Perseverative errors & 0.57 & $=0.46$ & 3.44 & $=0.008$ & 1.25 & $=0.30$ & 3.57 & $=0.834$ & 18.44 & $<0.0001$ & 2.40 & $=0.359$ \\
\hline
\end{tabular}

ANOVA results are reported for the effect of mPFC lesion, the effect of cue duration or delay duration, and the relevant interactions. Significant results $(P<$ $0.05)$ are highlighted in gray. 
A
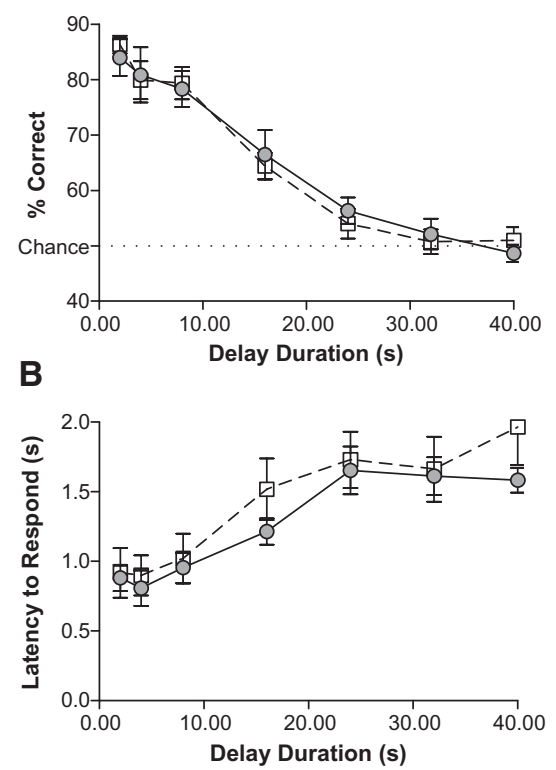

t) Sham

-o- mPFC Lesion

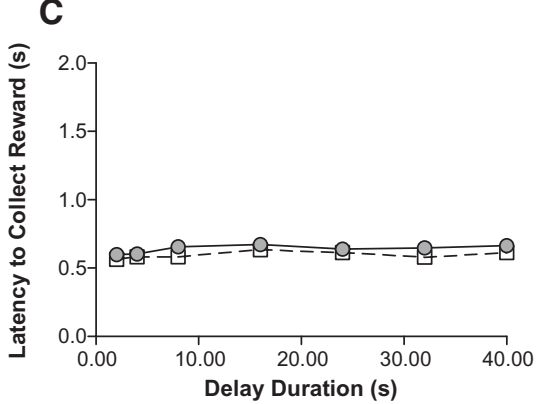

Figure 3. Lesion of the mouse mPFC does not impact performance on a working memory maintenance task. No significant difference was found between the lesioned and control subjects for percent correct responses $(A)$, latency to respond on a lever $(B)$, and latency to collect the milk reward $(C)$, averaged across three daily sessions for each delay duration tested. $n=7$ per group.

As with the attention task, we analyzed response latency, latency to collect rewards, rewards retrieved, omitted trials, perseverative responses, and perseverative errors. We found no effect of lesion on any of these behavioral measures (Table 1). Previous studies suggest that rats may use mediating motor behaviors to rehearse response choice during delay intervals in matching or nonmatching to sample operant tasks similar to the one we present here (Herremans et al. 1996; Chudasama and Muir 1997). However, there are no published data demonstrating that performance of any particular mediating motor behavior during delay intervals improves response accuracy in operant tasks. We observed three to four mice per condition throughout the experiment and did not observe any obvious stereotyped behavior.

In the present study, we held task requirements constant while assaying specific cognitive processes. Our adaptation of a simple visual discrimination task enabled us to test general learning ability and acquisition of the basic discrimination task (within $6 \mathrm{wk}$ ) and then test both sustained attention and working memory maintenance (within $6 \mathrm{wk}$ ), all while maintaining the same experimental design, with identical task rules, motivation, and response requirements. All that varied between the tasks was the duration of the cue presentation or the interval between cue and choice presentation. With this approach, we have determined the impact of mPFC lesions in mice on sustained attention and working memory maintenance.

mPFC lesions in mice reduced accuracy on the version of the task designed to assay sustained attention, concordant with evidence that PFC lesions affect sustained attention in rats (Muir 1996; Chudasama and Muir 2001; Passetti et al. 2002). In these studies, rats with mPFC lesions also showed an increase in perseverative responding. Similarly, we observed a trend for an increase in perseverative responding, though this did not reach significance. We observed no impact of mPFC lesion on the delayed choice version of the task. Because we tested the mice on the memory version of the task after the attention version, we cannot exclude the possibility that the animals experienced some func- tional recovery over the course of the experiment, leading to a false dissociation of attention and memory after PFC lesion. However, the ibotenic acid injections resulted in permanent neuronal loss, as evidenced by microscopic analysis of nissl-stained brain sections prepared after all behavioral testing was completed. It is not likely that the mice learned to compensate for the lesion because, after completing the attention task, subjects were tested again with the 5-sec cue duration, 1-sec delay condition, to retrain to stable discrimination performance. We did not see a difference in accuracy on this condition at the beginning of the experiment compared to the last day of retraining before starting the memory version of the task 3 wk later. Additionally, a dissociation between attention and mnemonic impairments has previously been shown in rats with dorsal PFC lesions using the lengthier CAM test (Chudasama et al. 2005).

Our finding that mPFC lesions in mice do not disrupt working memory maintenance is consistent with the evidence showing that DLPFC damage in humans has no impact on the maintenance of information in working memory but rather impacts the ability to manipulate information in working memory (Muller et al. 2002; D'Esposito et al. 2006). It is also consistent with the extensive fMRI literature demonstrating that activity in the DLPFC in humans is associated with the manipulation of information during working memory tasks but not the maintenance of information during delay intervals (Owen et al. 1996; D’Esposito et al. 1999; for review, see Wager and Smith 2003). Such validation of shared functional anatomy is critical for the successful use of animal models in studying cognitive functions that are disrupted in human patient populations.

The behavioral assays used in our present study allowed us to dissociate two distinct, yet often coupled, cognitive processes: sustained attention and working memory maintenance. This technique of using a single-task construct with discrete manipulations to isolate specific behavioral processes can be extended to test many other cognitive functions. For example, discrete manipulations of a visual discrimination task could be used to assay the components of working memory not tested here, such as resistance to distraction or memory capacity. Such an approach provides greater possibilities for relating specific components of cognitive functions to their underlying biological processes. Attempts to model cognitive endophenotypes of psychiatric disorders would especially benefit from this methodology, as it would allow investigators to focus on the key specific cognitive functions that are disrupted in patients.

\section{Acknowledgments}

This work was supported by National Institute of Mental Health Grant R01MH068073 (P.D.B.); the National Institute of Mental Health Silvio O. Conte Center for Schizophrenia Research MH086404 (E.H.S., E.R.K.); National Institute of Mental Health Grant F32MH090750-01 (R.D.W.); the Lieber Institute for Brain Development (R.D.W., E.R.K.); and the Howard Hughes Medical Institute (E.R.K., L.W.K.). We thank Iram Haq for maintaining the mouse colony. 


\section{References}

Aggleton JP, Neave N, Nagle S, Sahgal A. 1995. A comparison of the effects of medial prefrontal, cingulate cortex, and cingulum bundle lesions on tests of spatial memory: Evidence of a double dissociation between frontal and cingulum bundle contributions. J Neurosci 15: 7270-7281.

Awh E, Jonides J. 2001. Overlapping mechanisms of attention and spatial working memory. Trends Cogn Sci 5: 119-126.

Baddeley A. 1992. Working memory. Science 255: 556-559.

Barch DM, Smith E. 2008. The cognitive neuroscience of working memory: Relevance to CNTRICS and schizophrenia. Biol Psychiatry 64: 11-17.

Brito GN, Brito LS. 1990. Septohippocampal system and the prelimbic sector of frontal cortex: A neuropsychological battery analysis in the rat. Behav Brain Res 36: 127-146.

Chudasama Y. 2011. Animal models of prefrontal-executive function. Behav Neurosci 125: 327-343.

Chudasama Y, Muir JL. 1997. A behavioural analysis of the delayed non-matching to position task: The effects of scopolamine, lesions of the fornix and of the prelimbic region on mediating behaviours by rats. Psychopharmacology (Berl) 134: 73-82.

Chudasama Y, Muir JL. 2001. Visual attention in the rat: A role for the prelimbic cortex and thalamic nuclei? Behav Neurosci 115: 417-428.

Chudasama Y, Robbins TW. 2004. Dopaminergic modulation of visual attention and working memory in the rodent prefrontal cortex. Neuropsychopharmacology 29: 1628-1636.

Chudasama Y, Nathwani F, Robbins TW. 2005. D-Amphetamine remediates attentional performance in rats with dorsal prefrontal lesions. Behav Brain Res 158: $97-107$.

Dalley JW, Cardinal RN, Robbins TW. 2004. Prefrontal executive and cognitive functions in rodents: Neural and neurochemical substrates. Neurosci Biobehav Rev 28: 771-784.

D'Esposito M, Postle BR, Ballard D, Lease J. 1999. Maintenance versus manipulation of information held in working memory: An event-related fMRI study. Brain Cogn 41: 66-86.

D'Esposito M, Cooney JW, Gazzaley A, Gibbs SE, Postle BR. 2006. Is the prefrontal cortex necessary for delay task performance? Evidence from lesion and FMRI data. J Int Neuropsychol Soc 12: 248-260.

Furster JM. 1997. Animal neurophysiology. In The prefrontal cortex: Anatomy, physiology and neuropsychology of the frontal lobe, pp. 66-102. Lippincott-Raven, New York.

Granon S, Vidal C, Thinus-Blanc C, Changeux JP, Poucet B. 1994. Working memory, response selection, and effortful processing in rats with medial prefrontal lesions. Behav Neurosci 108: 883-891.

Harvey PO, Le Bastard G, Pochon JB, Levy R, Allilaire JF, Dubois B, Fossati P. 2004. Executive functions and updating of the contents of working memory in unipolar depression. J Psychiatr Res 38: 567-576.

Herremans AH, Hijzen TH, Welborn PF, Olivier B, Slangen JL. 1996. Effects of infusion of cholinergic drugs into the prefrontal cortex area on delayed matching to position performance in the rat. Brain Res 711: $102-111$.
Muir JL. 1996. Attention and stimulus processing in the rat. Brain Res Cogn Brain Res 3: 215-225.

Muller NG, Machado L, Knight RT. 2002. Contributions of subregions of the prefrontal cortex to working memory: Evidence from brain lesions in humans. J Cogn Neurosci 14: 673-686.

Ongur D, Price JL. 2000. The organization of networks within the orbital and medial prefrontal cortex of rats, monkeys and humans. Cereb Cortex 10: $206-219$.

Owen AM, Evans AC, Petrides M. 1996. Evidence for a two-stage model of spatial working memory processing within the lateral frontal cortex: A positron emission tomography study. Cereb Cortex 6: $31-38$.

Park S, Holzman PS. 1992. Schizophrenics show spatial working memory deficits. Arch Gen Psychiatry 49: 975-982.

Passetti F, Chudasama Y, Robbins TW. 2002. The frontal cortex of the rat and visual attentional performance: Dissociable functions of distinct medial prefrontal subregions. Cereb Cortex 12: 1254-1268.

Ragozzino ME, Adams S, Kesner RP. 1998. Differential involvement of the dorsal anterior cingulate and prelimbic-infralimbic areas of the rodent prefrontal cortex in spatial working memory. Behav Neurosci 112: $293-303$.

Research Domain Criteria Project. 2010. In Working memory: Workshop proceedings, Bethesda, MD. http://www.nimh.nih.gov/researchfunding/rdoc/working-memory-workshop.pdf.

Rose EJ, Ebmeier KP. 2006. Pattern of impaired working memory during major depression. J Affect Disord 90: 149-161.

Seamans JK, Floresco SB, Phillips AG. 1995. Functional differences between the prelimbic and anterior cingulate regions of the rat prefrontal cortex. Behav Neurosci 109: 1063-1073.

Taylor CL, Latimer MP, Winn P. 2003. Impaired delayed spatial win-shift behaviour on the eight arm radial maze following excitotoxic lesions of the medial prefrontal cortex in the rat. Behav Brain Res 147: $107-114$.

Wager TD, Smith EE. 2003. Neuroimaging studies of working memory: A meta-analysis. Cogn Affect Behav Neurosci 3: 255-274.

Ward RD, Simpson EH, Kandel ER, Balsam PD. 2011. Modeling motivational deficits in mouse models of schizophrenia: Behavior analysis as a guide for neuroscience. Behav Processes 87: $149-156$.

Ward RD, Kellendonk C, Kandel ER, Balsam PD. 2012a. Timing as a window on cognition in schizophrenia. Neuropharmacology 62: 1175-1181.

Ward RD, Simpson EH, Richards VL, Deo G, Taylor K, Glendinning JI, Kandel ER, Balsam PD. 2012b. Dissociation of hedonic reaction to reward and incentive motivation in an animal model of the negative symptoms of schizophrenia. Neuropsychopharmacology 37: 1699-1707.

Received March 8, 2012; accepted in revised form July 12, 2012. 


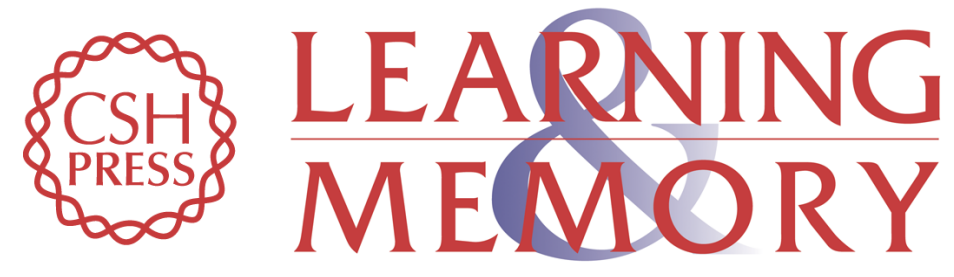

\section{Medial prefrontal lesions in mice impair sustained attention but spare maintenance of information in working memory}

Julia B. Kahn, Ryan D. Ward, Lora W. Kahn, et al.

Learn. Mem. 2012, 19:

Access the most recent version at doi:10.1101/Im.026302.112

Supplemental http://learnmem.cshlp.org/content/suppl/2012/10/16/19.11.513.DC1
Material

References This article cites 30 articles, 2 of which can be accessed free at: http://learnmem.cshlp.org/content/19/11/513.full.html\#ref-list-1

License

Email Alerting Receive free email alerts when new articles cite this article - sign up in the box at the Service top right corner of the article or click here. 